\title{
Mass Spectrometric Detection and Reductive Degradation of the Anti-cancer Drugs Ifosfamide and Cyclophosphamide
}

\author{
C. Laauser ${ }^{1}$, G. Habekost ${ }^{2}$, A. Habekost ${ }^{1,}$ \\ ${ }^{1}$ Ludwigsburg University of Education, Department of Chemistry, Reuteallee 46, D-71634 Ludwigsburg, Germany \\ ${ }^{2}$ University of Hildesheim, Hildesheim, Germany \\ *Corresponding author: A.Habekost@t-online.de
}

\begin{abstract}
A flow tube reactor containing iron or zinc powder in a nitrogen atmosphere is capable of performing $>90 \%$ anaerobic reductive dehalogenation of the cancer drugs ifosfamide and cyclophosphamide at $400{ }^{\circ} \mathrm{C}$ using toluene, alcohol or water as proton donors and iron or zinc as reducing agents. The products are all chlorine-free, as proven by GC-MS. The process is particularly interesting for large-scale industrial applications.
\end{abstract}

Keywords: reductive dehalogenation, anti-cancer drugs, mass spectrometry

Cite This Article: C. Laauser, G. Habekost, and A. Habekost, "Mass Spectrometric Detection and Reductive Degradation of the Anti-cancer Drugs Ifosfamide and Cyclophosphamide." Applied Ecology and Environmental Sciences, vol. 6, no. 1 (2018): 15-22. doi: 10.12691/aees-6-1-3.

\section{Introduction}

\subsection{Ifosfamide and Cyclophosphamide as Anti-cancer Drugs}

Chemotherapy's ability to kill cancer cells depends on its ability to stop cell division. Such drugs usually work by damaging the RNA or DNA that tells a cell how to copy itself when dividing. If the cells cannot divide, they will die. The faster the cells divide, the more likely it is that chemotherapy can kill the cells and cause the tumor to shrink. Alkylating agents are also most active during a cell's resting phase.

Unfortunately, chemotherapy cannot differentiate between cancerous cells and normal cells. The cells most frequently affected by chemotherapy are blood cells and cells in the mouth, stomach, intestine and hair follicles.

After the USA entered World War II, two young American pharmacologists, Louis Goodman and Alfred Gilman, investigated the influence of nitrogen mustards on mice and rabbits. To test the toxicity of these nitrogen mustards, they injected several substances that had already produced during the First World War. N-Lost (N,N-Bis(2-chlorethyl)-N-methylamin) was able to significantly reduce tumor growth. In addition, they found that the blood of soldiers contaminated with N-Lost who survived a poisonous gas attack had a significantly lower leukocyte concentration [1].

Ifosfamide and cyclophosphamide are chemotherapeutic agents. Ifosfamide and cyclophosphamide are chemically related to N-Lost (Figure 1).

Both substances are used today, often in combination with epirubicin, to cure various forms of (pediatric) sarcoma and cancer [2].

All anti-cancer drugs are harmful, because they have toxic effects on humans. Ifosfamide and cyclophosphamide have serious urotoxic side effects because they metabolize to 4-hydroxy-ifosfamide, 4-hydroxy-cyclophosphamide, acrolein and phosphoramide mustard, all known to be toxic substances [3]. Most of these substances are synthesized via biotransformation of ifosfamide and cyclophosphamide in the liver (Figure 2).

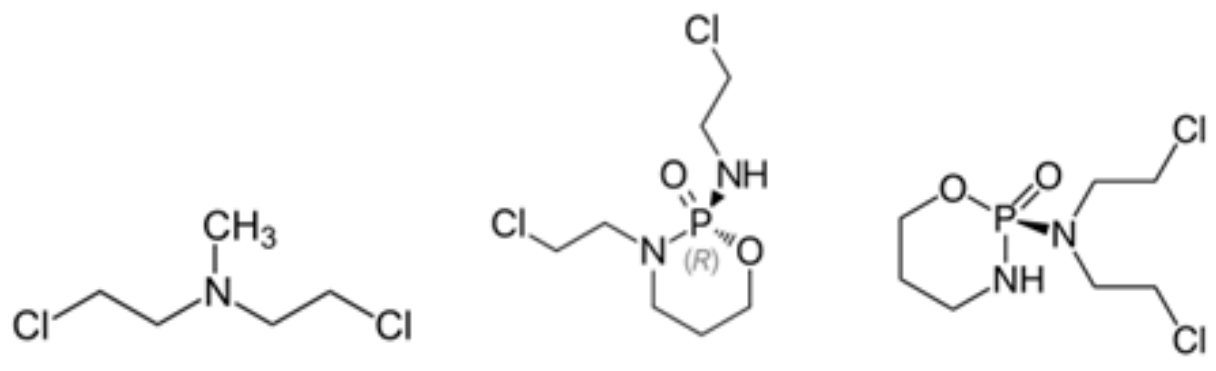

Figure 1. N-Lost, ifosfamide, cyclophosphamide 


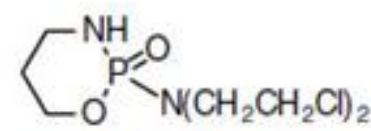

cyclophosphamide

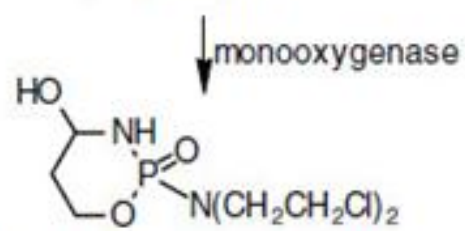

4-hydroxy-cyclophosphamide

$\downarrow$

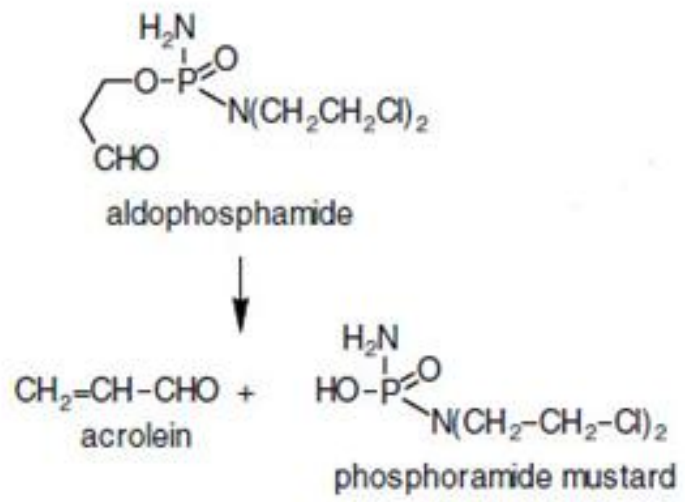

Figure 2. Part of the biotransformation of cyclophosphamide [4]

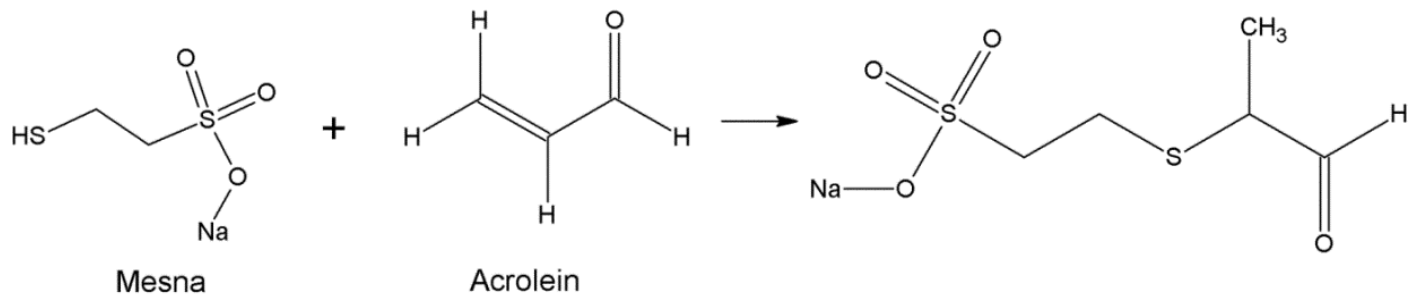

Figure 3. Reaction between mesna and acrolein

Mesna (2-mercaptoethanesulfonate $\mathrm{C}_{2} \mathrm{H}_{5} \mathrm{NaO}_{3} \mathrm{~S}_{2}$ ) prevents ifosfamide- or cyclophoshamide-induced hemorrhagic cystitis, bleeding and irritation of the bladder by binding acrolein to the sulfhydryl moiety in a thiol-ene reaction (Michael addition) (Figure 3).

Several thiol and thio compounds have been tested as active agents to prevent the urotoxicity of ifosfamide and cyclophosphamide [5]. In this context, MacAllister et al. [6] found that carbonyl or amine scavengers offer more protection against chloroacetaldehyde than against acrolein (both substances cause protein damage due to the degradation of glutathione).

\subsection{Ifosfamide and Cyclophosphamide as Pollutants - Waste Water Treatments}

A high percentage of cancer drugs enter the aquatic environment and persist without significant biodegradation. As a rule, normal waste water treatment cannot detoxify these substances. Additional procedures are therefore required to remove or degrade them.

Zhang et al. [7] investigated the fate and ecotoxicology of various cytostatic drugs in the environment such as alkylating agents, antimetabolites, plant alkaloids, hormones and antagonists and other substances such as cisplatin. These substances come mainly from hospitals and household waste water (especially urinary excretion). The household contamination was found to increase drastically over the last years. The authors examined different methods of removing cytostatic drugs, such as degradation in a bioreactor, different kinds of osmosis filtration and advanced oxidation processes. However, they were unable to determine the best method.

Cesen et al. [8] reported on biological and abiotic treatments of ifosfamide and cyclophosphamide. The highest separation efficiency was $59 \%$ for cyclophosphamide and
$35 \%$ for ifosfamide. The rate of removal in advanced oxidation processes (ozonization and / or UV, hygrodynamic cavitation) was significantly higher.

One such process is photodegradation in the presence of $\mathrm{UV} / \mathrm{H}_{2} \mathrm{O}_{2}, \mathrm{UV} / \mathrm{Fe}^{2+} / \mathrm{H}_{2} \mathrm{O}_{2}$ or $\mathrm{UV} / \mathrm{TiO}_{2}$ [9]. A variant of this experiment is a simulated sunlight treatment as a means of improving biodegradability [10]. The authors summarized that all these processes are effective in removing cyclophosphamide, but none of them achieved complete degradation.

The UV/ $/ \mathrm{Fe}^{2+} / \mathrm{H}_{2} \mathrm{O}_{2}$ system exhibited the highest degradation rate, with $76 \%$ of cyclophosphamide mineralized within 2 minutes. The products were analyzed using HPLC tandem mass spectrometry and the degree of mineralization was measured using a TOC analyzer. The luminescent bacteria strain Vibrio fischeri was chosen to test the toxicity of degradation products.

Lin and Lin [11] eliminated $99.9 \%$ of cyclophosphamide within 4 hours, but they observed that only $79.9 \%$ was dechlorinated and only $55.1 \%$ was mineralized after 16 hours of irradiation. The authors suggested that the oxidation products of cyclophosphamide are more recalcitrant and toxic than cyclophosphamide itself. This was measured with a Microtox analyzer, which is also based on the bioluminescence of $V$. fischeri.

A cyclic voltammogram of ifosfamide in acetonitrile with lithium perchlorate as the supporting electrolyte shows two irreversible anodic peaks at 1.85 and $2.00 \mathrm{~V}$ (vs a saturated calomel electrode). Both processes are two-electron processes. Electrochemical oxidation of ifosfamide and cyclophosphamide leads to 4-methoxy-ifosfamide and 4-methoxy-cyclophosphamide [12]. The methoxylized oxidation products have a much higher cytotoxicity than the reactants. Fabianska et al. [13] measured the degradation of ifosfamide and cyclophosphamide with a boron-doped diamond thin film electrode and found a significant influence of the initial concentration and the 
current density. In addition, $\mathrm{Cl}^{-}$- and $\mathrm{PO}_{4}{ }^{2-}$ - ions significantly accelerate the degradation of ifosfamide. Since the $\mathrm{C}_{2} \mathrm{H}_{4} \mathrm{Cl}$ side chains of ifosfamide and cyclophosphamide are linked with different and identical $\mathrm{N}$-atoms, the electrolytic intermediate degradation products of ifosfamide and cyclophosphamide were different.

Recently, Prasad et al. functionalized a screen-printed electrode (SPE) based on graphene quantum dots [14]. The differential pulse anodic stripping signal is about 7 times higher than that of the non-functionalized graphene SPE. The limit of detection for ifosfamide was about $0.11 \mathrm{ng} / \mathrm{mL}$ $(\mathrm{S} / \mathrm{N}=3)$.

Our goal is the complete detoxification of ifosfamide and cyclophosphamide in a simple, low-cost flow or batch reactor under anaerobic conditions with iron or zinc powder.

\section{Experiments}

Chemicals and instruments: Ifosfamide (Sigma, 14909), cyclophosphamide monohydrate (Sigma, C0768), iron (1-10 $\mu \mathrm{m}$, Merck 1.03819), zinc $(<150 \mu \mathrm{m}$, Sigma Aldrich 324930).
GCMSD (GC: Hewlett-Packard 6890 with HP 5 column; temperature program: start temperature: $50^{\circ} \mathrm{C}, 2 \mathrm{~min}$ isotherm; temperature rate: $15^{\circ} \mathrm{C} / \mathrm{min}$; final temperature: $270^{\circ} \mathrm{C}, 2 \mathrm{~min}$ isotherm), MSD: Hewlett-Packard 5973, injection: $1 \mu \mathrm{L})$.

\subsection{Characterization of Ifosfamide and Cyclophosphamide Using GCMS}

Ifosfamide and cyclophosphamide can be analyzed conventionally together using GC-MSD with sufficient resolution (see Figure 4).

Cyclophosphamide is partially degraded during the GC analysis by cleavage of $\mathrm{HCl}$ and cyclization peak $\mathrm{D}$ in Figure 4).

Although the masses are identical $(261 \mathrm{u})$, the mass spectra of ifosfamide (Figure 5, top) and cyclophosphamide (Figure 5, bottom) differ significantly.

The loss of $\mathrm{Cl}(175 \mathrm{amu})$ is much more pronounced in cyclophosphamide, whereas the loss of $\mathrm{C}_{2} \mathrm{H}_{4} \mathrm{Cl}$ / $\mathrm{C}_{2} \mathrm{H}_{4} \mathrm{CINH}$ (70 amu), the loss of $\mathrm{C}_{2} \mathrm{H}_{4} \mathrm{ClNH}$ (134 amu) and the loss of $\mathrm{C}_{2} \mathrm{H}_{4} \mathrm{NHN}$ (154 amu) are more significant in ifosfamide.

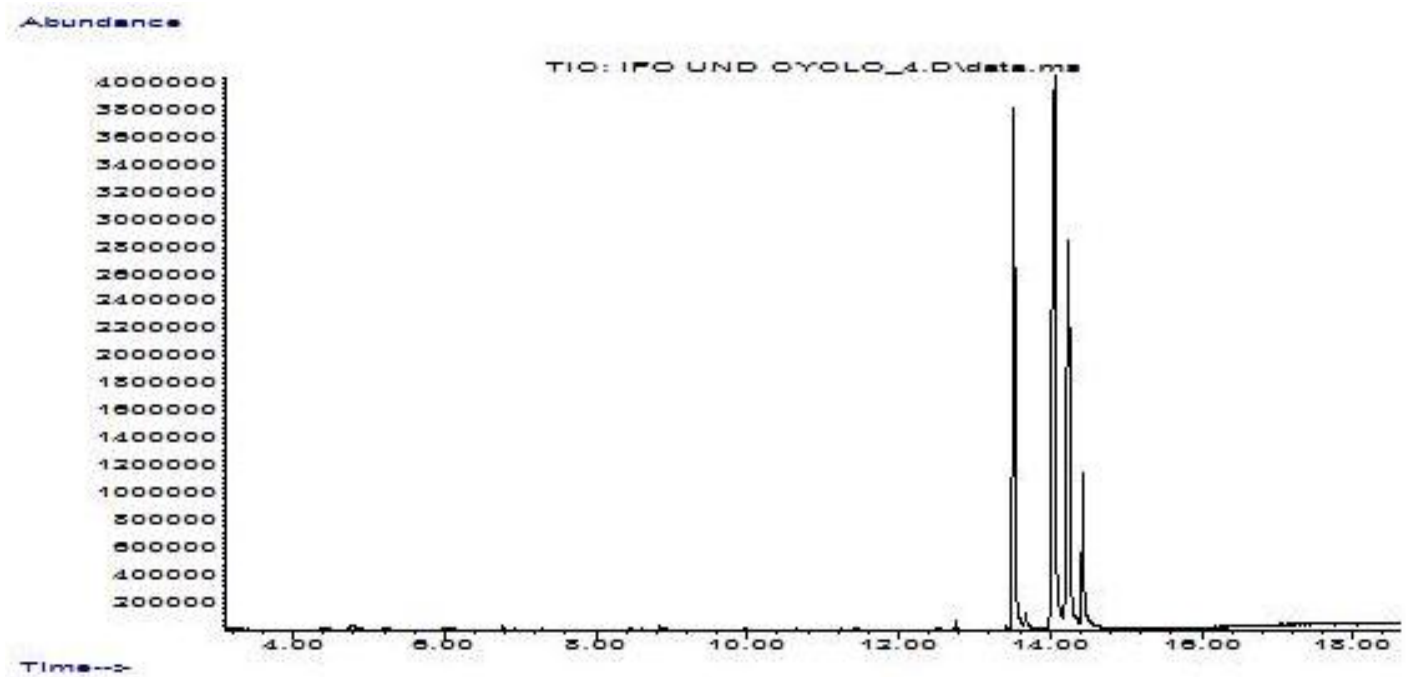

Figure 4. GCMSD of ifosfamide and cyclophosphamide together: A, B: cyclophosphamide, C: ifosfamide, D: degradation product of cyclophosphamide resulting from the $\mathrm{GC}$-analysis (dissociation of $\mathrm{HCl}$ and cyclization)
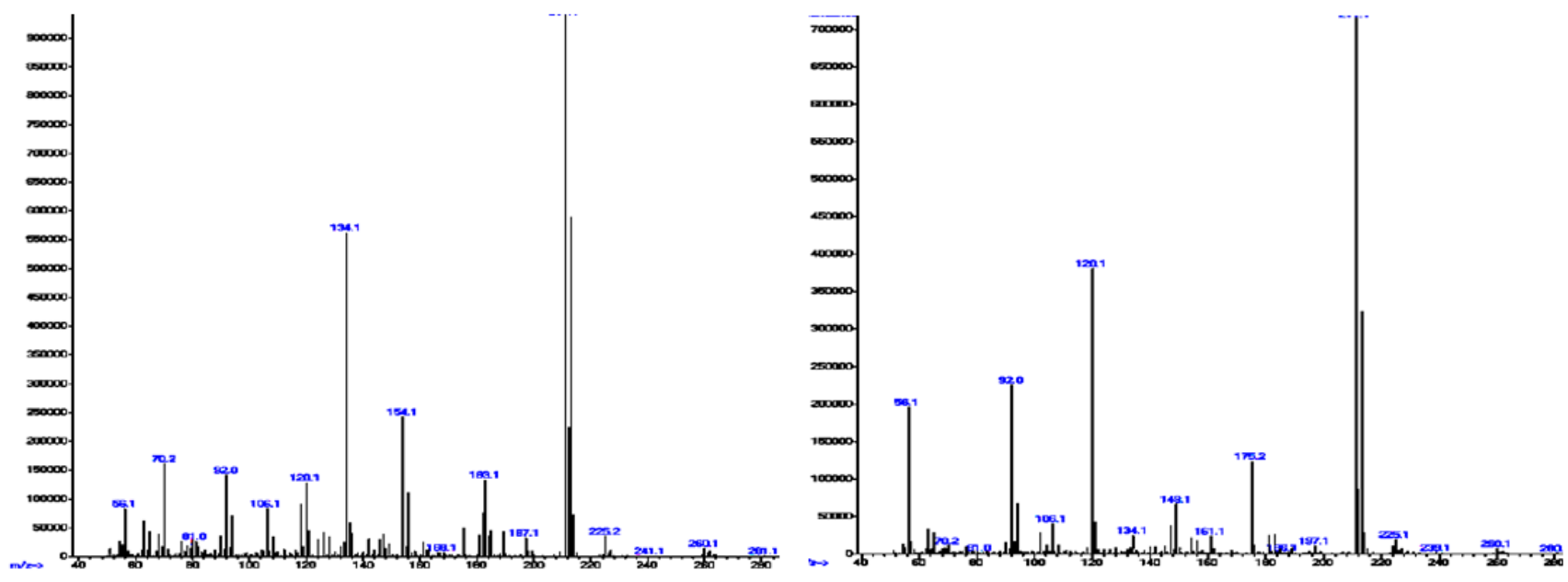

Figure 5. Mass spectra of Ifoasfamide (top), cyclophosphamide (bottom) 

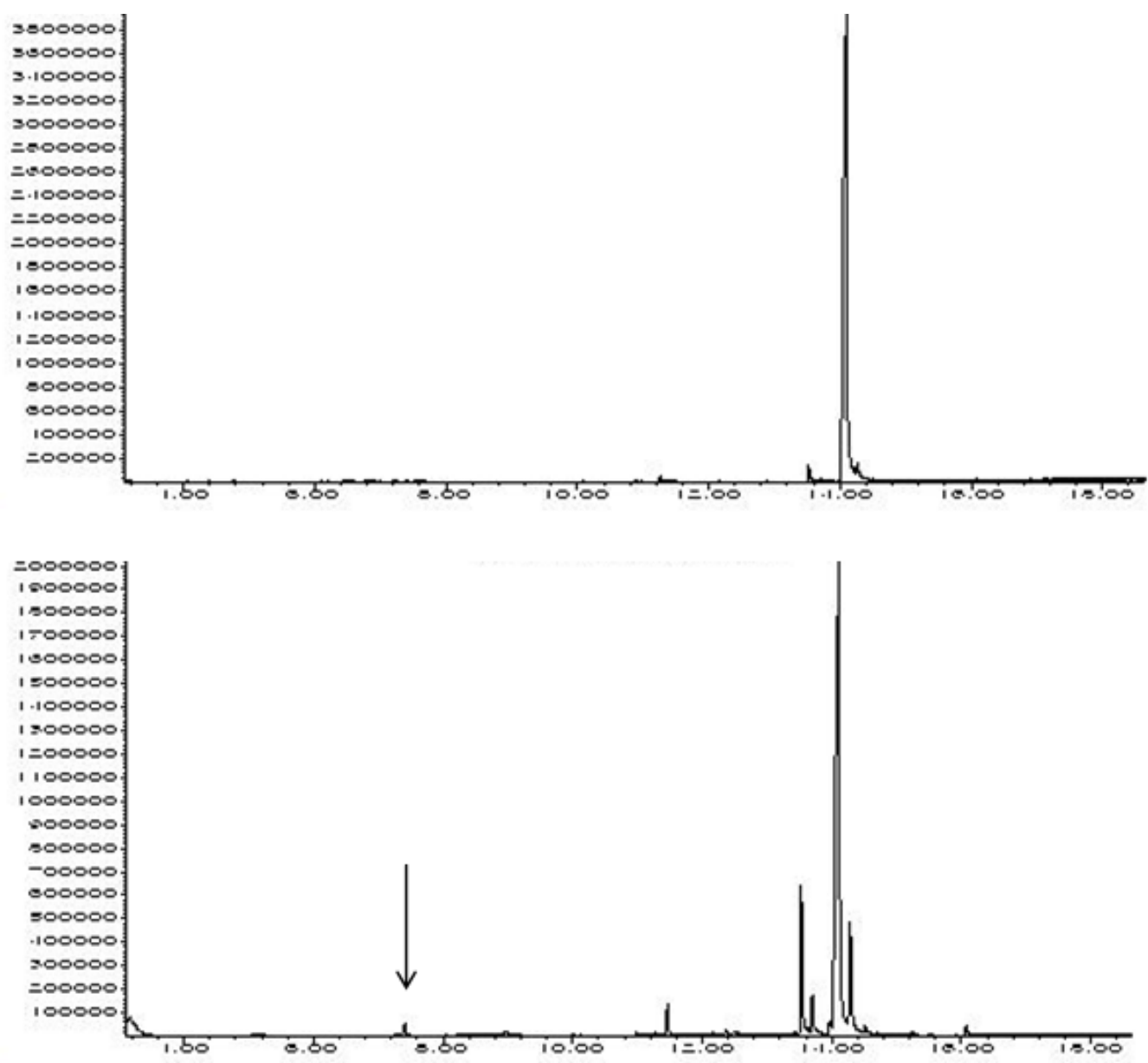

Figure 6. Gas chromatogram of ifosfamide before (top) and after irradiation with daylight for $1 \mathrm{~h}$ (bottom)

Irradiation with daylight can damage ifosfamide (and cyclophosphamide, not illustrated). Figure 6 shows the GC of ifosfamide without (top) and after irradiation with daylight (bottom, irradiation time: $1 \mathrm{~h}$ ). Ifosfamide is partially degraded to N-Lost (see arrow in Figure 6). The other degradation products are not identifiable.

Therefore, in chemotherapeutics ifosfamide solutions must be protected from daylight.

\subsection{Reductive Dehalogenation of Ifosfamide and Cyclophosphamide with Zero-valent Iron and Zinc}

Our goal is to develop a technical method to completely remove ifosfamide and cyclophosphamide using dehalogenation reagents, and which is not energy intensive. The dehalogenation reagent must be inexpensive, and the end products must be benign.

Organochloride contaminants can be detoxified by simple chemical substitution of the chlorine atoms $[15,16]$. Each Brønsted acid can be used as a hydrogen source for the reduction reaction. However, in order to prevent reaction with the dehalogenating reagent, it is important to choose a weak acid.

We tested three different proton sources: toluene, propanol and water. The main advantage of water is that the contaminated waste water itself can be treated directly. However, ifosfamide and cyclophosphamide are more soluble in propanol and toluene, which in turn increases the effectiveness of total degradation after extraction of ifosfamide and cyclophosphamide. Another advantage of using organic solvents is that other organic contaminants can also be easily decomposed after extraction.

In theory, almost any non-noble metal could be used to make a metal chloride. Alkalis and alkali earths would be the most active reducing agents, but their high reactivity is disadvantageous here. Due to their rapid oxidation in air or moist media, they can only be used in an inert atmosphere or in inert solvents. Working in a nitrogen atmosphere to avoid this problem is precluded because of the proclivity of these metals to form nitrides at high temperatures. For this reason, we examined the possibilities offered by the less reductive iron and zinc powders.

\section{Materials and Methods}

The reactor was a V2A stainless steel tube $18 \mathrm{~mm}$ in diameter and $700 \mathrm{~mm}$ long. About $20 \mathrm{~g}$ of iron powder (or zinc powder) was gently mixed with a $0.5 \mathrm{~g}$ tuft of glass wool in a rotary mixer until a homogeneous, fluffy powder was obtained. This was poured into the tube over a length of about $200 \mathrm{~mm}$. The reactor was heated with a hinged tube furnace (Carbolite, HST 12-400).

The operating mode of the reactor is designed to allow injection of condensed phase reactants under controlled, prolonged, continuous flow conditions. In tests with toluene, propanol or water as the proton donor, ifosfamide and cyclophosphamide and toluene (propanol, water) were 
mixed in a separate vessel in a mass ratio of 1:50 and then transferred to a $1-\mathrm{ml}$ syringe. The liquid mixture was then injected into the reactor tube for about $10 \mathrm{~s}$ and vaporized inside it.

A nitrogen gas stream $\left(\mathrm{N}_{2}\right.$, purity $5.0,10 \mathrm{~mL} / \mathrm{min}$ flow rate) transports the gaseous reactants and dehalogenation products through the tube. After leaving the tube, the products are condensed in a cooled trap, and then brought to an off-line GC/MS for analysis (via micro-syringe extraction).

Another, simpler batch setup consists of a three-necked flask with a reflux condenser, the flask is filled with the zero-valent iron or zinc powder. The solution of ifosfamide or cyclophosphamide in toluene, propanol or water is injected through a syringe into the heated flask (at different temperature). The temperature was heated by a controlled heating system and regulated via a thermocouple.

After 5 minutes of intense stirring, the flask was thoroughly rinsed with additional ether (ether comprises butylhydroxytoluene BHT as stabilizing agent, which is used as internal standard in GC).
The gas chromatograms (Figure 7) show a significant reduction in ifosfamide at temperatures above $300^{\circ} \mathrm{C}$. $400^{\circ} \mathrm{C}$ is required to achieve the desired goal of "complete" conversion: at these temperatures, a comparison of peak heights shows that conversion rates of better than $90 \%$ are achieved.

It is difficult to identify the peaks detected (we used several MS databases: NBS, Wiley and NIST). But, as Figure 7 shows, the ifosfamide peak decreases as a function of temperature. An analysis of the mass spectra shows that most peaks are due to toluene (see Figure 7, top) but do not contain chlorine. However, exact identification of the peaks was not easy, despite the use of the aforementioned mass spectra databases.

Ultimately, the lifetime of the dehalogenation reagent the iron or zinc powder - was determined using stoichiometry. The dehalogenation process stops when the metallic iron or zinc reagent has been completely converted to iron or zinc chlorides and, in all likelihood, also iron or zinc carbides (not tested here, but tested in previous studies $[15,16])$.

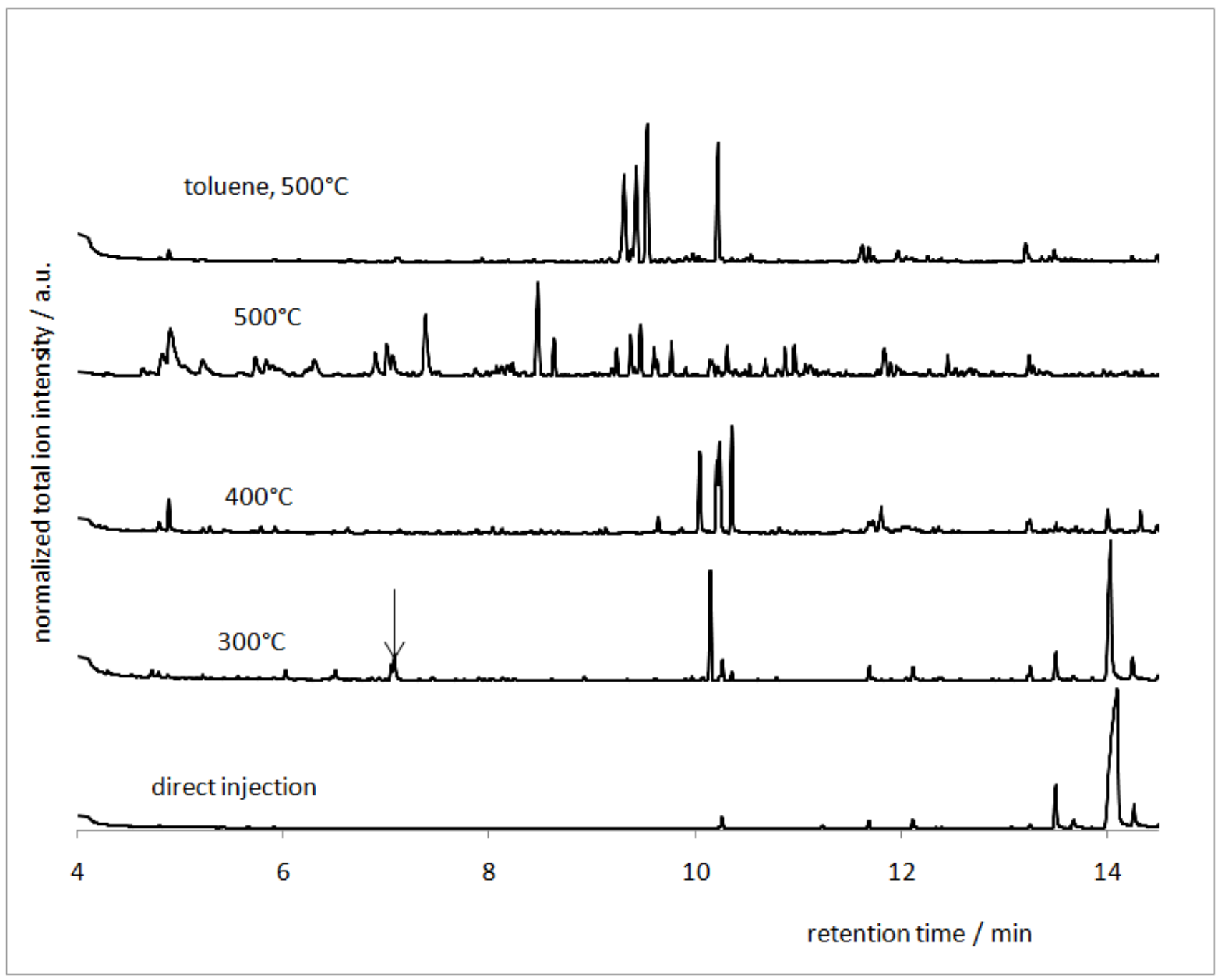

Figure 7. GC of ifosfamide (retention time: $14 \mathrm{~min}$ ) at different dehalogenation temperatures. At $300^{\circ} \mathrm{C}$, N-Lost (retention time at about 7 min, see arrow) is one of the degradation products. Proton source: toluene. Reductant: iron. Top: solvent toluene at $500^{\circ} \mathrm{C}$

The $\mathrm{GC}$ at $300^{\circ} \mathrm{C}$ shows that one of the degradation products is $\mathrm{N}$-Lost (retention time 7 min., see arrow). However, $\mathrm{N}$ Lost degrades at higher temperatures.

With propanol as the proton source, the degradation of ifosfamide was analogous, but the degradation of propanol was less pronounced. 


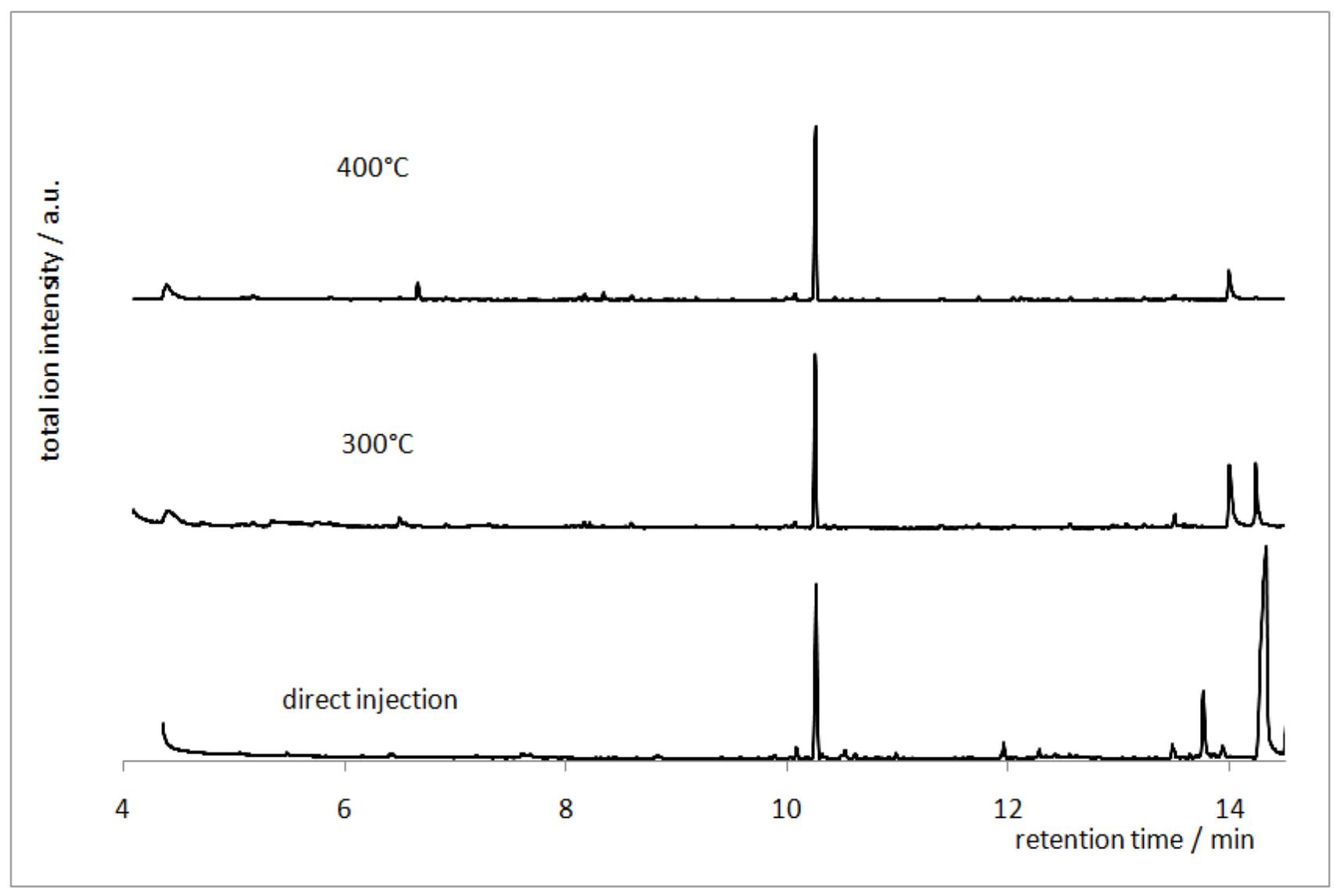

Figure 8. GC of ifosfamide at two different dehalogenation temperatures with iron as reductant and n-propanol as proton source. BHT as internal standard (retention time $10.5 \mathrm{~min}$ )

With zinc as reductant, the degradation of ifosfamide was more effective. At $300^{\circ} \mathrm{C}$, around $93 \%$ of the ifosfamide had already degraded.

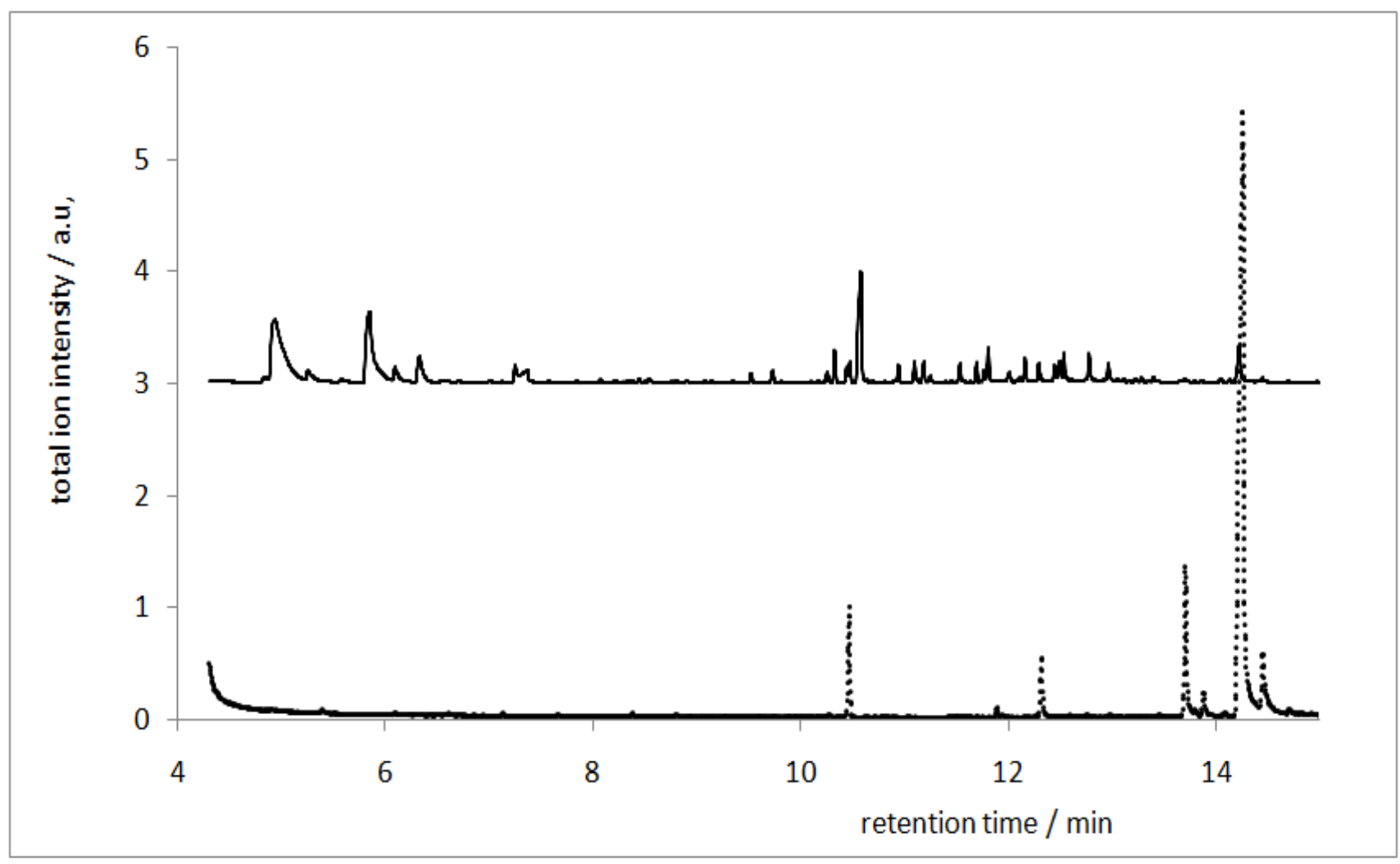

Figure 9. GC of ifosfamide at $300^{\circ} \mathrm{C}$ with zinc as reductant and toluene as proton source. BHT as internal standard. GC is normalized to the BHT intensity 
Table 1. Comparison between iron and zinc powder in the degradation of ifosfamide with toluene as solvent

\begin{tabular}{|c|c|c|c|c|}
\hline temperature & $\begin{array}{c}\text { degradation rate of } \\
\text { ifosfamide with iron as } \\
\text { reductant }\end{array}$ & $\begin{array}{c}\text { degradation rate of } \\
\text { cyclophosphamide with iron as } \\
\text { reductant }\end{array}$ & $\begin{array}{c}\text { degradation rate of } \\
\text { ifosfamide with zinc as } \\
\text { reductant }\end{array}$ & $\begin{array}{c}\text { degradation rate of } \\
\text { cyclophosphamide with zinc as } \\
\text { reductant }\end{array}$ \\
\hline $300^{\circ} \mathrm{C}$ & $10 \%$ & not measured & $93 \%$ & $76 \%$ \\
\hline $400^{\circ} \mathrm{C}$ & $84 \%$ & $85 \%$ & $100 \%$ & $100 \%$ \\
\hline $500^{\circ} \mathrm{C}$ & $96 \%$ & $95 \%$ & $100 \%$ & $100 \%$ \\
\hline
\end{tabular}

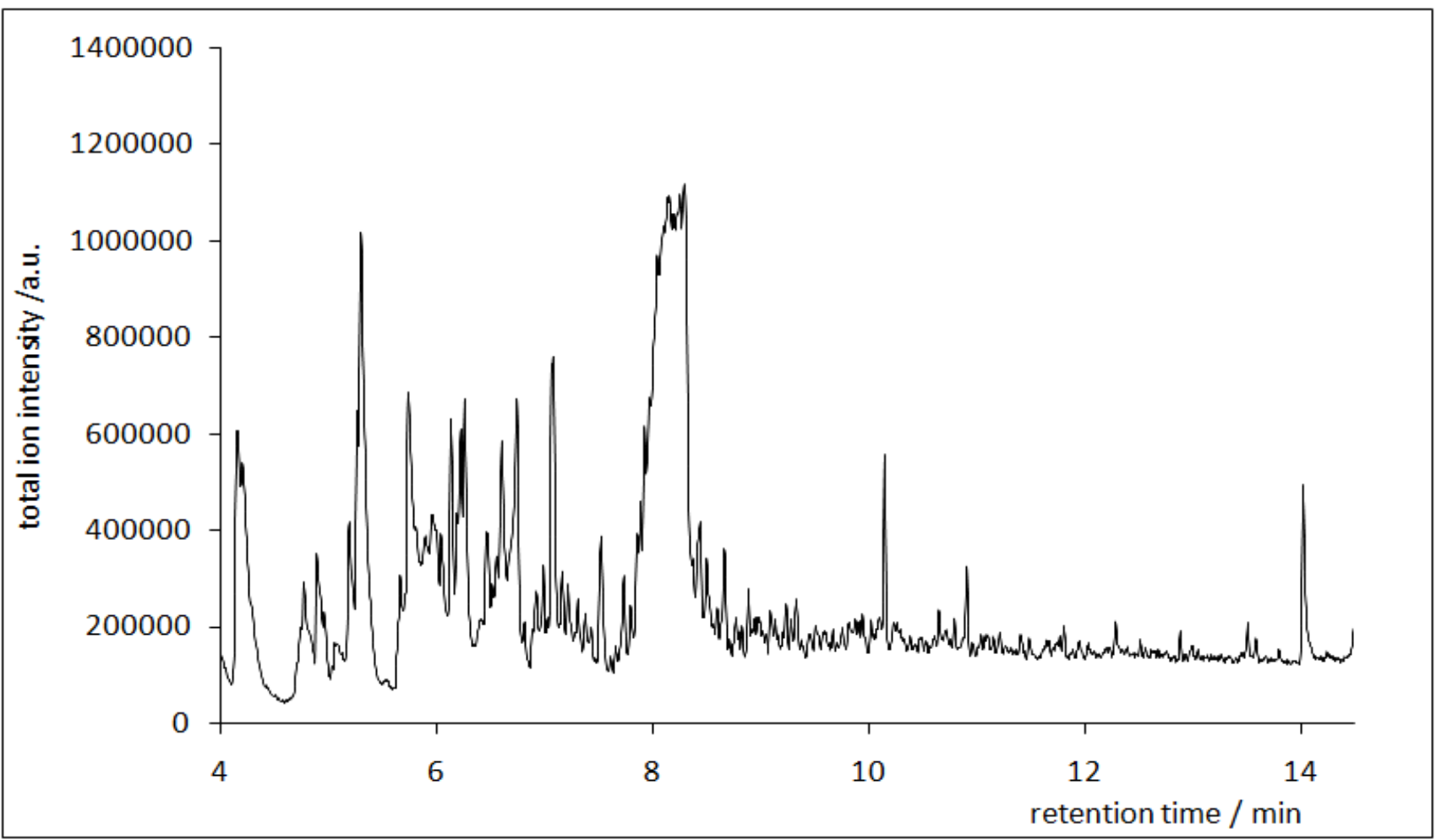

Figure 10. GC of ifosfamide after aerobic heating without an additional proton source

Table 1 compares the degradation yield of ifosfamide and cyclophosphamide with iron and zinc powder at different temperatures with toluene as proton source.

Using water as proton source also produced a significant degradation of ifosfamide at $400^{\circ} \mathrm{C}(89 \%$ degradation with zinc and $32 \%$ with iron). Therefore, from a technical perspective, the simple thermal degradation of waste water containing ifosfamide especially with zinc will be successful at high temperatures. In contrast to zinc, iron needs higher temperatures. We think that water reacts with iron to form iron oxide / hydroxide which partially prevents the reduction of ifosfamide.

Aerobic heating of ifosfamide without an additional proton source results in a complex product distribution: The main peak is probably a derivate of N-Lost (but not $\mathrm{N}$-Lost itself), because the fragmentation pattern shows masses (106 u, $92 \mathrm{u}, 78 \mathrm{u}, 63 \mathrm{u})$ which can be assigned to N-Lost.

Therefore, simple aerobic heating of the substances alone is not an effective technique.

\subsection{Extraction of Ifosfamide and Cyclophosphamide from Soil}

\subsubsection{Chemicals and Materials}

Ifosfamide and cyclophosphamide, ethanol, separatory funnel, Soxhlet extractor, rotary evaporator

\subsubsection{Experimental Procedure}

We dissolved $20 \mathrm{mg}$ ifosfamide (cyclophosphamide) in $10 \mathrm{~mL}$ ethanol and a GCMS was recorded as reference. The solution was then mixed with $15 \mathrm{~g}$ dried soil and extracted with $100 \mathrm{~mL}$ ethanol in a Soxhlet extractor for $2 \mathrm{~h}$. The extract was then concentrated with a rotary evaporator, dried in a drying oven at $70^{\circ} \mathrm{C}$ for two hours, and analyzed with GCMS after adding exact $10 \mathrm{~mL}$ ethanol. This was the probe.

The quotient of integrals of the ifosfamide (cyclophosphamide) GC peaks with and without soil was: $\mathrm{I}_{\text {probe }} / \mathrm{I}_{\text {reference }}$ (ifosfamide) $\approx 95 \%$ recovery rate, $\mathrm{I}_{\text {probe }} /$ $\mathrm{I}_{\text {reference }}($ cyclophosphamide $) \approx 95 \%$ recovery rate - a quite acceptable result.

\section{Conclusion and Further Work}

Although the iron or zinc reductants are very promising for use in water remediation, some questions remain unanswered. First, we found that it was possible to completely convert the substances using just the empty steel tube without iron powder inside, albeit at higher temperatures (above $600^{\circ} \mathrm{C}$ ). Reducing the iron powder grain size (e.g., to nanometer scales) will presumably enable efficient dehalogenation at even lower temperatures than necessary here. Second, tests should be conducted replacing the nitrogen carrier gas with air. 
The main problem is finding an effective and pollutionfree method to extract ifosfamide and cyclophosphamide from waste water and finding reductants that do not corrode in the presence of water. Iron alloys such as ironchromium or iron-nickel alloys may be an attractive alternative.

\section{Acknowledgements}

A. H. thanks the Vector Foundation (Germany), the Fonds der Chemischen Industrie (Germany), and the Ludwigsburg University of Education for financial support.

\section{References}

[1] https://ptaforum.pharmazeutische-zeitung.de/index.php?id=5977.

[2] S. Frustaci, S. Foladore, A. Buonadonna, A. De Paoli, D Crivellari, A. Carbone, R. Sorio, S. Morassut, Epirubicin and ifosfamide in advanced soft tissue sarcomas, Ann Oncol. 4, 669-72, 1993.

[3] M. Mohrmann, S. Ansorge, U. Schmich, B. Schönfeld, M. Brandis, Toxicity of ifosfamide, cyclophosphamide and their metabolites in renal tubular cells in culture, Pediatr Nephrol. 8, 157-163, 1994.

[4] http://onlinelibrary.wiley.com/doi/10.1002/3527600418.mb10702 e0016/pdf.

[5] N. Brock, J. Pohl, J. Stekar, Studies on the Urotoxicity of Oxazaphosphorine Cytostatics and its Prevention. 2. Comparative studiy on the Uorprotective Efficacy of Thiols and other Sulfur Compounds, Eur. J. Cancer Clin. Oncol. 17, 1155-1163, 1981.

[6] S. MacAllister, N. Martin-Brisac, V. Lau, K. Yang, P.J. O’Brien, Acrolein and chloroacetaldehyde: An examination of the cell and cell-free biomarkers of toxicity, Chemico-Biological Interactions 202, 259-266, 2013.
[7] J. Zhang, V.W.C. Chang, A. Giannis, J-Y. Wang, Removal of cyctostatic drugs from aquatic environment: A review, Science of the Total Environment, 445-446, 281-298, 2013.

[8] M. Cesen, T. Kosjek, M. Laimou-Geraniou, B. Kompare, B. Sirok, D. Lambropolou, E. Heath, Occurrence of cyclophosphamide and ifosfamide in aqueous environment and their removal by biological and abiotic wastewater, Science of the Total Environment, 527-528, 465-473, 2015.

[9] C.A. Lutterbeck, E.L. Machado, K. Kümmerer, Photodegradation of the antineoplastic cyclophosphamide: A comparative study of the efficiencies of $\mathrm{UV} / \mathrm{H}_{2} \mathrm{O}_{2}, \mathrm{UV} / \mathrm{Fe}^{2+} / \mathrm{H}_{2} \mathrm{O}_{2}$ or $\mathrm{UV} / \mathrm{TiO} \mathrm{O}_{2}$ processes, Chemosphere, 120, 538-546, 2015.

[10] C.A. Lutterbeck, M.L. Wilde, E. Baginska, C. Leder, E.L. Machado, K. Kümmerer, Degradation of cyclophosphamide and 5-fluorouracil by $U V$ and simulated sunlight treatments: Assessment of the enhancement of the biodegradability and toxicity, Environm. Poll. 208, 467-476, 2016.

[11] H.H-H. Lin, A. Y-C. Lin, Photocatalytic oxidation of 5fluorouracil and cyclophosphamide via $\mathrm{UV}_{\mathrm{TiO}}$ in an aqueous environment, Wat. Res. 48, 559-568, 2014.

[12] A. Paci, T. Martens, J. Royer, Anodic Oxidation of Ifosfamide and Cyclophosphamide: A Biomimetic Metabolism Model of the Oxazaphosphorinane Anticancer Drugs, Bioorganic \& Medicinal Chem. Lett. 11, 1347-1349, 2001.

[13] A. Fabianska, A. Ofiarska, A. Fiszka-Borzyszkowska, P. Stepnowski, E.M. Siedlecka, Electrodegradation of ifosfamide and cyclophosphamide at BDD electrode: Decomposition pathway and its kinetics, Chem. Engin. Journal 276, 274-282, 2015.

[14] B.B. Prasad, A. Kumar, R. Singh, Synthesis of novel monomeric graphene quantum dots and corresponding nanocomposite with molecularly imprinted polymer for electrochemical detection of an anticancerous ifosfamide drug, Biosensors and Bioelectronics 94, $1-9,2017$.

[15] N. Aristov, A. Habekost, Heterogeneous dehalogenation of PCBs with iron/toluene or iron/quicklime, Chemosphere, 80, 113-115, 2010.

[16] A. Habekost, N. Aristov, Heterogeneous reductive dehalogenation of PCB contaminated transformer oil and brominated diphenyl ethers with zero valent iron, Chemosphere, 88, 1283-1287, 2012. 